\title{
Utilidade da Cintilografia Miocárdica na Avaliação de Pacientes Submetidos à Revascularização Percutânea: Importância de Seguir as Diretrizes
}

\author{
Ver artigo relacionado \\ na página 107
}

\author{
Gabriel Blacher Grossman ${ }^{1,2}$
}

A cintilografia miocárdica (CM) é um método de imgem bem estabelecido na prática cardiológica e a sua utilidade para a tomada de decisões e conduta encontra respaldo em farta literatura publicada nas duas últimas décadas ${ }^{1}$. O uso da técnica tomográfica (SPECT - single photon emission computed tomography), de agentes marcados com Tc-99m, da análise semiquantitativa por meio de escores de gravidade, da análise quantitativa dos defeitos perfusionais com uso de base de dados de pacientes normais e da análise funcional do ventrículo esquerdo sincronizada com o eletrocardiograma incrementou a sua acurácia diagnóstica. O valor diagnóstico e prognóstico da CM na avaliação de pacientes com cardiopatia isquêmica suspeita ou estabelecida está bem documentado em diversos cenários clínicos, como na avaliação de dor torácica, na estratificação de risco após síndrome coronariana aguda, na avaliação terapêutica e na pesquisa de viabilidade miocárdica.

Outro cenário clínico em que a CM é comumente utilizada é na avaliação de pacientes que foram submetidos à revascularização percutânea (RP). A CM pode demonstrar o sucesso do procedimento, evidenciando redução da área isquêmica, bem como avaliar de maneira adequada aqueles pacientes que apresentam dor torácica após a revascularização e estratificar o risco de eventos cardíacos após o procedimento ${ }^{2}$. Foi demonstrado que a sensibilidade do teste ergométrico é inferior à $\mathrm{CM}$ na avaliação de pacientes submetidos à RP ${ }^{1}$. A anormalidade perfusional após a revascularização pode corresponder a complicações peri-procedimento, ao comprometimento de ramos secundários coronarianos, à isquemia em artérias não revascularizadas, à reestenose do vaso tratado ou à nova obstrução. Cabe ressaltar que foi demonstrado que a presença de sintomas nem sempre é um bom marcador de reestenose, já que até $25 \%$ dos pacientes assintomáticos podem apresentar isquemia após a realização de angioplastia e colocação de stent $^{3,4}$.

Recentemente, foram publicadas as indicações para a utilização clínica da CM, sendo a avaliação de pacientes após RP um dos cenários clínicos analisados ${ }^{5}$. Em pacientes sintomáticos após revascularização, o uso da CM na avaliação da etiologia da dor é considerado apropriado. Em pacientes assintomáticos, quando o indivíduo não apresentava sintomas antes do procedimento, a utilização da CM para detecção de isquemia e avaliação terapêutica é considerada válida, sendo necessários mais dados na literatura para uma recomendação definitiva. No entanto, se o paciente está assintomático e apresentava sintomas antes da revascularização, o uso da CM em um período inferior a dois anos é considerado inadequado. Nesta última situação, quando o período for superior a dois anos, o uso da CM poderá ser considerado, embora as opiniões dos especialistas não sejam consensuais. As diretrizes do Grupo de Estudos de Cardiologia Nuclear/Sociedade Brasileira de Cardiologia não recomendam o uso rotineiro da CM em pacientes assintomáticos após $\mathrm{RP}^{6}$.

Outra situação que merece discussão é a avaliação precoce de pacientes após RP. As diretrizes americanas sugerem que não se faça estudo cintilográfico nos primeiros 2 meses após o procedimento'. Isto porque alguns estudos demonstraram que outros fatores, que não reestenose, podem alterar a perfusão miocárdica nas primeiras semanas após a RP. Esta questão é analisada no artigo de Marin-Neto et al. ${ }^{7}$, nesta edição da $\mathrm{RBCl}$. Neste estudo, os autores analisaram 36 pacientes que foram randomizados para tratamento com angioplastia coronariana ou aterectomia rotacional, com evidência cintilográfica de isquemia miocárdica e lesão

\footnotetext{
${ }^{1}$ Cardionuclear - Instituto de Cardiologia, Porto Alegre, RS.

${ }^{2}$ Serviço de Medicina Nuclear do Hospital Moinhos de Vento, Porto Alegre, RS.

Correspondência: Gabriel Blacher Grossman. Cardionuclear - Av. Princesa Isabel, 395 - Porto Alegre, RS, Brasil - CEP $90620-001$.

Fone: (51) 3217-2580 - Fax: (51) 3217-7200 • E-mail: ggrossman@terra.com.br

Recebido em: 07/05/2007 - Aceito em: 08/05/2007
} 
coronariana $\geq 70 \%$. Em um período médio de sete dias após a intervenção, os pacientes foram submetidos à nova CM. O índice de sucesso clínico e angiográfico foi similar nos dois grupos $(93 \%$ no grupo que realizou aterectomia vs. 95\% no grupo submetido à angioplastia). Os valores de duplo produto, no momento da injeção do radiofármaco, também foram similares. Os autores descrevem uma redução das alterações perfusionais ao estresse, em ambos os grupos, mas com significância estatística no grupo da angioplastia. No estudo em repouso, houve redução do defeito perfusional, no grupo da angioplastia, e aumento da hipoperfusão, no grupo submetido à aterectomia (sem significância estatística). A conclusão do estudo é que as anormalidades perfusionais precoces após o procedimento estejam relacionadas a distúrbios microcirculatórios associados à microembolizações durante a aterectomia.

Os achados do estudo de Marin-Neto et al. ${ }^{7}$ são similares aos resultados de diversos estudos já publicados na literatura, demonstrando alterações perfusionais em pacientes que realizam precocemente a CM após RP. Koch et al. ${ }^{8}$ demonstraram alta incidência de defeitos perfusionais transitórios com a realização de CM até dois dias após aterectomia, em pacientes com reestenose intra-stent ou novas lesões (88 e 92\%, respectivamente). No entanto, a incidência, a extensão e a gravidade da hipoperfusão não se relacionaram com o tipo ou quantidade do material retirado, sugerindo que a embolização da placa não seja o principal causador dos defeitos perfusionais, quando comparado com outros fatores, como agregação plaquetária ou microcavitações. Este mesmo autor demonstrou redução da incidência, extensão e gravidade da hipoperfusão transitória pós-aterectomia com o uso do bloqueador da GPIIb/IIla, o que sugere que a agregação plaquetária, realmente, tenha papel importante na etiologia das alterações perfusionais precoces após RP com aterectomia ${ }^{9}$. Em outro estudo que analisou pacientes após angioplastia ou colocação de stent, os achados perfusionais na CM, nas primeiras 24 horas do procedimento, não predizeram a necessidade de nova revascularização $\operatorname{tardia}^{10}$. Outro aspecto importante a ser considerado é o trauma e provável disfunção endotelial associada como causa de alterações perfusionais transitórias. Sabese, à luz da evidência atual, que alterações cintilográficas em pacientes com vasos epicárdicos sem lesões hemodinamicamente significativas podem estar relacionadas à redução da reserva coronariana, demonstrada, por exemplo, com quantificação absoluta de fluxo coronariano por meio da tomografia por emissão de pósitrons ${ }^{11}$. Pacientes diabéticos podem apresentar alterações perfusionais e funcionais na $\mathrm{CM}$, angiografia com artérias coronárias normais e teste não invasivo demonstrando disfunção endotelial (por exemplo, teste da reatividade da artéria braquial) ${ }^{12}$. Embora não existam dados suficientes na literatura sobre este tema, o trauma e a disfunção endotelial transitória represen- tam uma explicação atraente para defeitos perfusionais precoces após RP.

Em contrapartida ao que foi demonstrado por MarinNeto et al. ${ }^{7}$, outros autores evidenciaram relação entre as alterações perfusionais precoces na CM e complicações peri-procedimento ou aumento da taxa de reestenose $\mathrm{P}^{13,14}$. A discrepância entre os achados angiográfico e cintilográfico também pode ser justificada por lesões não analisadas adequadamente pela angiografia. Bachmann et al. ${ }^{15}$ demonstraram que, nesta situação, a ultra-sonografia coronária pode ser útil, ao evidenciar uma maior freqüência de lesão residual nos pacientes com isquemia 48 horas após procedimento de revascularização $(50 \%$ de estenose em pacientes com CM anormal vs. 33\% em pacientes com CM normal).

Apesar do tamanho da amostra do estudo de Marin-Neto et al. ${ }^{7}$ ser pequeno, nenhum estudo que avaliou a questão apresentou uma população com um número significativamente maior de pacientes. O estudo é consistente em demonstrar resultados já previamente descritos na literatura e ressalta a importância da relação de outros fatores que não reestenose nas alterações perfusionais precoces após aterectomia. Mesmo não sendo evidenciado neste trabalho, a literatura demonstra que a CM realizada precocemente após angioplastia/stent também pode demonstrar alterações perfusionais que não estão relacionadas à reestenose e, provavelmente, sejam causadas por algum dos fatores já discutidos previamente. Este é um dado significativo, tendo em vista que a aterectomia não é um procedimento de RP realizado freqüentemente em nosso meio. É importante que estudos futuros avaliem a freqüência das alterações perfusionais precoces em pacientes submetidos a técnicas de RP mais recentes e que realizem nova CM em um período mais longo de acompanhamento, para determinar se houve ou não resolução do defeito perfusional observado, bem como uma angiografia de controle para correlação dos dados angiográficos e cintilográficos.

Na prática clínica, qual a importância destes achados? Deve-se reconhecer que alterações cintilográficas observadas em CM realizada precocemente após RP (até dois meses) não necessariamente estão relacionadas à reestenose. No entanto, esta alteração deve ser valorizada quando o paciente apresentar sintomas. A utilização da CM após RP, de acordo com as diretrizes estabelecidas, diminui a chance de valorizar achados pouco específicos e permite selecionar um grupo de pacientes em que a CM pode fornecer informações perfusionais e funcionais importantes que auxiliem no manejo do paciente revascularizado.

\section{REFERÊNCIAS BIBLIOGRÁFICAS}

1. Klocke FJ, Baird MG, Lorell BH, Bateman TM, Messer JV, Berman DS, et al. ACC/AHA/ASNC guidelines for the clinical use of cardiac radionuclide imaging-executive summary: a report of the American College of Cardiology/American Heart Association Task Force on Practice Guidelines (ACC) 
AHA/ASNC Committee to Revise the 1995 Guidelines for the Clinical Use of Cardiac Radionuclide Imaging). Circulation. 2003;108(11):1404-18.

2. Ho KT, Miller TD, Holmes DR, Hodge DO, Gibbons RJ. Long-term prognostic value of Duke treadmill score and exercise thallium-201 imaging performed one to three years after percutaneous transluminal coronary angioplasty. Am J Cardiol. 1999;84(11):1323-7.

3. Hecht HS, Shaw RE, Chin HL, Ryan C, Stertzer SH, Myler RK. Silent ischemia after coronary angioplasty: evaluation of restenosis and extent of ischemia in asymptomatic patients by tomographic thallium-201 exercise imaging and comparison with symptomatic patients. J Am Coll Cardiol. 1991;17(3): 670-7.

4. Marie PY, Danchin N, Karcher G, Grentzinger A, Juilliere $\mathrm{Y}$, Olivier $\mathrm{P}$, et al. Usefulness of exercise SPECT-thallium to detect asymptomatic restenosis in patients who had angina before coronary angioplasty. Am Heart J. 1993;126(3 Pt 1):571-7.

5. Brindis RG, Douglas PS, Hendel RC, Peterson ED, Wolk MJ, Allen JM, et al. ACCF/ASNC appropriateness criteria for singlephoton emission computed tomography myocardial perfusion imaging (SPECT MPI): a report of the American College of Cardiology Foundation Quality Strategic Directions Committee Appropriateness Criteria Working Group and the American Society of Nuclear Cardiology endorsed by the American Heart Association. J Am Coll Cardiol. 2005;46(8): 1587-605.

6. Sociedade Brasileira de Cardiologia. Atualização da Diretriz da Sociedade Brasileira de Cardiologia sobre Cardiologia Nuclear. Arq Bras Cardiol. 2006;86(Supl.1).

7. Marin-Neto JA, Lima-Filho MO, Simões MV, Pintya AO, Horta PE, Ariê S. Persistência de distúrbios perfusionais miocárdicos após intervenção coronária percutânea com êxito: dependência de fatores microcirculatórios. Rev Bras Cardiol Invas. 2007;15(2):107-14.
8. Koch KC, Radke PW, Kleinhans E, Ninnemann S, Janssens $\mathrm{U}$, Klues HG, et al. Mechanisms of myocardial hypoperfusion during rotational atherectomy of de novo coronary artery lesions and stenosed coronary stents: insights from serial myocardial scintigraphy. J Nucl Cardiol. 2002;9(3):304-11.

9. Koch KC, vom Dahl J, Kleinhans E, Klues HG, Radke PW, Ninnemann S, et al. Influence of a platelet GPIIb/IIla receptor antagonist on myocardial hypoperfusion during rotational atherectomy as assessed by myocardial Tc-99m sestamibi scintigraphy. J Am Coll Cardiol. 1999;33(4):998-1004.

10. Jaffe R, Haim SB, Karkabi B, Front A, Gips S, Weisz G, et al. Myocardial perfusion abnormalities early (12-24h) after coronary stenting or balloon angioplasty: implications regarding pathophysiology and late clinical outcome. Cardiology. 2002;98(1-2):60-6.

11. Iskandrian AE. The Achilles heel of SPECT imaging: the falsepositive scans-or are they? J Nucl Cardiol. 2006;13(6):747-8.

12. Papaioannou GI, Kasapis C, Seip RL, Grey NJ, Katten D, Wackers FJ, et al. Value of peripheral vascular endothelial function in the detection of relative myocardial ischemia in asymptomatic type 2 diabetic patients who underwent myocardial perfusion imaging. J Nucl Cardiol. 2006;13(3):362-8.

13. Iskandrian AS, Lemlek J, Ogilby JD, Untereker WJ, Cave V, Heo J. Early thallium imaging after percutaneous transluminal coronary angioplasty: tomographic evaluation during adenosine-induced coronary hyperemia. J Nucl Med. 1992;33(12): 2086-9.

14. Rodes-Cabau J, Candell-Riera J, Domingo E, Castell-Conesa J, Anivarro I, Angel J, et al. Frequency and clinical significance of myocardial ischemia detected early after coronary stent implantation. J Nucl Med. 2001;42(12):1768-72.

15. Bachmann R, Sechtem U, Voth E, Voth E, Hopp HW, Schicha $\mathrm{H}$. Dipyridamole scintigraphy and intravascular ultrasound after successful coronary intervention. J Nucl Med. 1997;38(4): 553-8. 